\title{
Local interstellar cosmic-ray spectra derived from gamma-ray emissivities
}

\author{
A. W. Strong*, on behalf of the Fermi-LAT Collaboration \\ Max-Planck-Institut für extraterrestrische Physik \\ E-mail: aws@mpe.mpg.de
}

Precise gamma-ray emissivities from cosmic-ray interactions with interstellar gas have been recently derived using Fermi-LAT data, and used to constrain the local interstellar spectra of protons and leptons. We report on a continuing effort to exploit these emissivities combined with the latest hadronic gamma-ray production cross-sections and other constraints such as synchrotron emission for the leptonic component. The interstellar spectra provide important information for heliospheric modulation, and cosmic-ray origin and propagation.

The 34th International Cosmic Ray Conference,

30 July- 6 August, 2015

The Hague, The Netherlands

${ }^{*}$ Speaker. 


\section{Introduction}

The spectrum of cosmic rays (CR) in the local interstellar medium (within about $1 \mathrm{kpc}$ from the Sun) is of interest as a complement to direct measurements and as a probe of solar modulation which affects particles below a few $\mathrm{GeV} . \gamma$-rays from $\mathrm{CR}$ protons and heavier nuclei interacting with interstellar gas are an ideal probe of local CR; bremsstrahlung from CR electrons and positrons is also important at low energies and must be accounted for too. In $(1 ; 2)$ we presented preliminary results based on earlier emissivity data (3). (4) addresses other aspects of this topic, also using those emissivities.

Recently a new and precise determination of the local $\gamma$-ray emissivity using Fermi-LAT data has been made (5), studying regions at Galactic latitudes out of the plane; in particular the contributions from atomic, molecular and ionized hydrogen were separated, and the emissivity of atomic gas traced by the 21-cm line can be used as the most reliable data for analysis. (5) also provided an extensive analysis of the emissivities to derive the interstellar proton spectum. Here we pursue this project with the new emissivities, with various innovations including new cross-sections and analysis techniques.

\section{Emissivity matrix representation}

\subsection{Response Matrices}

The observed emissivity is the sum of hadronic (pion-production) and leptonic (bremsstrahlung) contributions: $q_{t o t}\left(E_{\gamma}\right)=q_{\text {had }}\left(E_{\gamma}\right)+q_{l e p}\left(E_{\gamma}\right)$. The gamma-ray emissivity predicted for the input CR spectra can be written in matrix form $\varepsilon_{k}^{\text {pred }}=\Sigma_{\text {process }} \Sigma_{j} M_{j k} I_{j}(\vec{\theta})$. where $\varepsilon_{k}$ is the emissivity in the k'th energy bin, and $I_{j}(\vec{\theta})$ is the j'th momentum sample point of the CR spectrum model with parameters $\vec{\theta}$. The processes run over hadronic and leptonic components, with $I_{j}(\vec{\theta})$ including CR protons, helium, electrons and positrons. $M_{j k}$ can include the dispersion in the Fermi-LAT $\gamma$-ray energy measurements. The matrix $M_{j k}$ is pre-computed for a given binning of data and model, so that the response can be computed very fast for a large number of model parameters. The method is general and can be extended to include cosmic-ray direct measurements, synchrotron emissivities etc. Once the matrices and data are defined, the problem is a purely mathematical one.

\subsection{Energy dispersion}

The Fermi-LAT energy measurement has a precision of about $15 \%$, becoming worse at low energies. Accounting for the energy dispersion is especially important below $200 \mathrm{MeV}$, where photons are both lost from the measured range and gained from higher and lower energies, depending on the input spectrum. The energy dispersion is defined as $p\left(E_{\text {meas }} \mid E_{\text {true }}\right)$, where $E_{\text {true }}, E_{\text {meas }}$ are the true and measured $\gamma$-ray energies respectively. $p\left(E_{\text {meas }} \mid E_{\text {true }}\right)$ is obtained in matrix form using the Fermi-LAT Science Tools, for the same event selection and response as used to derive the emissivities. The approximation is made that the response averaged over the full sky is appropriate; the variations are small and average out making this a good approximation. The measured spectrum is also weighted by the Fermi-LAT effective area which is a function of energy, varying rapidly at low energies. The full transfer function from the model proton and helium spectra to measured 
emissivities in energy bands can be expressed as a single matrix, allowing fast computation for statistical analysis.

\section{Bayesian analysis}

The likelihood function is $L($ data $\mid \vec{\theta})=\exp \left(-\Sigma_{k}\left(\varepsilon_{k}^{\operatorname{pred}(\vec{\theta})}-\varepsilon_{k}^{o b s}\right)^{2} / 2 \sigma_{k}^{2}\right)$ where $\varepsilon_{k}^{o b s}$ is the observed emissivity and $\sigma_{k}$ is the error estimate for the k'th energy bin. By Bayes theorem the posterior probability of the model is $P(\vec{\theta} \mid$ data $)=\frac{P(\vec{\theta}) L(\text { data } \mid \vec{\theta})}{P(\text { data })}$ where $P(\vec{\theta})$ is the prior probability for the parameters $\vec{\theta}$ defining the model, and $P($ data) is a normalizing factor (known as the evidence ) such that $\int P(\vec{\theta} \mid$ data $) d^{N} \vec{\theta}=1$. Given the joint probability distribution of the parameters, we can marginalize to obtain the distribution of any subset, including single parameters: $P\left(\theta_{i}\right)=\int_{\theta \neq \theta_{i}} P(\vec{\theta} \mid$ data $) d^{N-1} \vec{\theta}$. Mean and standard deviations are $\bar{\theta}_{i}=\int_{\theta \neq \theta_{i}} \theta_{i} P(\vec{\theta} \mid$ data $) d^{N-1} \vec{\theta}$, $\sigma\left(\theta_{i}\right)=\sqrt{(} \int_{\theta \neq \theta_{i}}\left(\theta_{i}-\bar{\theta}_{i}\right)^{2} P(\vec{\theta} \mid$ data $\left.) d^{N-1} \vec{\theta}\right)$. Since we are actually more interested in the CR spectrum than in a particular set of parameters, which are anyway highly correlated, it is useful also to compute the mean and standard deviation of the gridded CR spectra $n_{j}=n\left(p_{j}\right)$ :

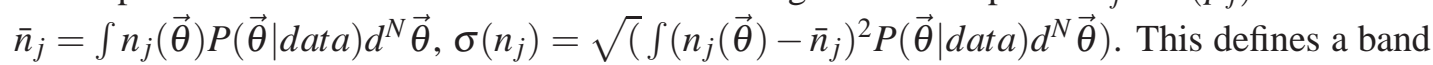
containing the range of CR spectra. This method is therefore a deconvolution using a basis of parameterized spectra ${ }^{1}$. The range of emissivities corresponding to this range can then be computed (for each process) for comparison with the observed values, to illustrate the uncertainties.

In our previous analysis $(1 ; 2)$ we used an explicit parameter scan, which was limiting due to the large number of parameters. The new analysis uses the MultiNest software ${ }^{2}$ (6), an advanced Bayesian package for multi-parameter fitting; among its advantages are that it does not require specification of a step-size, has handling of multi-modal posteriors, and allow computation of the statistics of functions of the parameters. We found it fast and reliable for the problem at hand. The ranges of the parameters have to be specified. As described below, we fit to eq.(6.1), and there are a total of 10 parameters to be fitted. The parameters in this application have rather limited ranges of reasonable values, and a flat prior over a prescribed range is sufficient for the present purpose; more complex priors are straightforward to include if required. MultiNest outputs full parameter chains, mean and standard deviations of each parameter, and mean and standard deviations of userdefined functions of the parameters, here chosen to be the formula representing the CR spectrum as specified above.

\section{4. $\gamma$-ray production functions}

Recent reviews of hadronic $\gamma$-ray production can be found in $(7 ; 1 ; 2 ; 4 ; 8)$. Here we use the QGSJET-II-04 model $(9 ; 7)$. In addition to photon production in proton-proton collisions, we have to account for the contribution to the photon yield by proton-helium, helium-proton, and heliumhelium interactions. Since QGSJET-II includes nuclei-nuclei interactions, the parametrization of (7) can be used directly for all four reaction channels above the transition energy. For $A>4$ we

\footnotetext{
${ }^{1}$ An alternative method, in principle preferable, would be to perform a parameter-free deconvolution of the CR spectra, with some regularizing prior; the present method is an intermediate approach.

${ }^{2}$ available at http://ccpforge.cse.rl.ac.uk/gf/project/multinest
} 
apply a nuclear enhancement factor following (8). For proton energies below $20 \mathrm{GeV}$ we use the $\gamma$-ray production functions described in $(1 ; 2)$; these are for proton-proton collisions only, so we again apply the factor given in (8) for p-He, He-p, He-He and heavier nuclei. The helium abundance of interstellar gas is 0.1 by number, and the interstellar gas composition for heavier nuclei uses (10). $\mathrm{CR}$ helium is taken into account explicitly, and the CR composition for heavier nuclei is based on ACE and CREAM. The contribution from CR and ISM with $\mathrm{A}>4$ is about $10 \%$ relative to $\mathrm{A} \leq 4$, and is dominated by $\mathrm{CR}$, much less coming from heavy gas nuclei.

Bremsstrahlung from CR leptons is computed using the corresponding GALPROP routine.

\section{Synchrotron and direct constraints on electrons}

Synchrotron emission from electrons and positrons in the interstellar magnetic field provides essential constraints on interstellar leptons, independent of solar modulation. For a survey of experimental data and theoretical arguments see $(11 ; 12 ; 13)$. We are not concerned here with the injection spectrum of electrons, or how the interstellar spectrum is affected by propagation, but just the observational information on the interstellar spectrum. As found in (11) the synchrotron brightness temperature spectral index $\beta$ changes from about 2.5 to 3 in the range from $100 \mathrm{MHz}$ to several GHz. The relation of $\beta$ to the electron index $\alpha$ is $\beta=2+(\alpha-1) / 2$, so this corresponds to a steepening of the interstellar electron spectrum from index 2 to 3 at a few $\mathrm{GeV}$ electron energy. At high energies and frequencies, this is consistent with the Fermi-LAT direct measurements of electrons + positrons, which give an index $3.08 \pm 0.05$ (14) and for electrons an index $3.19 \pm 0.07$ (15), the electrons fully dominating at low energies where bremsstrahlung is important; this is also consistent with microwave synchrotron emission measured with Planck (16). Here solar modulation is negligible so that this consistency is a requirement, assuming the local measurement is typical of the interstellar medium near the solar position in the Galaxy. At low energies, only synchrotron is a reliable tracer of the interstellar electron spectrum, due to the very large solar modulation (factor or 10 or more). The relation of synchrotron to the electron spectrum is a complicated function (11), but it is useful to write a simplified version to illustrate the nature of the constraints on the energy of the electron spectral index break. We use the formula from sec 2.1.1 and 2.3 of (11) and the synchrotron data from that paper. We have for the synchrotron peak frequency for a electron en-

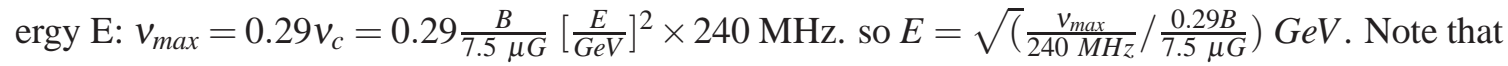
$\mathrm{E}$ depends on $\sqrt{v}_{\text {max }}$ and $\sqrt{B}$ so is robust against uncertainties in these quantities. The observed break $v$ is in the range $500-5000 \mathrm{MHz}$, B is between 5 and $10 \mu G$, using a broad conservative range. Hence taking extremes of $v / B, 2<E_{\text {break }}<10 \mathrm{GeV}$. A narrower range is also reasonable: $v=500-2000 \mathrm{MHz}, B=5-8 \mu G$, giving $2.6<E_{\text {break }}<6.6 \mathrm{GeV}$. The upper limit is consistent with Fermi-LAT electrons which show no break down to $7 \mathrm{GeV}$. However other experiments (AMS01, PAMELA) show the break must be below $3 \mathrm{GeV}$, since there is no break observed above this energy and solar modulation has the effect of enhancing the break in the directly measured spectrum. Hence the adopted break energy constraint from synchrotron and direct measurements is 1-2.5 GeV. The synchrotron constraint is essential for the low end and the index below the break. Since we use a smooth function for the break, the relation between the break parameter and the actual spectrum is not exact. We use the Fermi-LAT electron spectrum as measured for the spectrum above the break, including the Fermi-LAT normalization at $100 \mathrm{GeV}$ and index range 3.1-3.2. 
Below the break, the experimental range of $\beta$ is 2.4 to 2.6 , so from the above formula we use the range $\alpha=1.8-2.2$ as the constraint from synchrotron.

\section{Parameterization of spectra}

As explained in $(1 ; 2)$ we use a spectrum in the form of density per unit momentum $n(p)$, since this is expected to have a power-law shape in the theory of diffusive shock acceleration, and hence serves as a suitable basis form on which propagation and other effects are superimposed. The local emissivities require a turnover in the proton spectrum towards low energies. A possible parameterization of CR spectra has a single sharp break in the spectral index, and this is often used; it was used in for example in $(1 ; 2)$. However this is unphysical, and a smoother function is more appropriate. Hence smooth breaks in protons, helium and electrons are modelled; the functional form used is $n(p) \propto 1 /\left[\left(\frac{p}{p_{b r}}\right)^{\alpha_{1} / \delta}+\left(\frac{p}{p_{b r}}\right)^{\alpha_{2} / \delta}\right]^{\delta}$ where $\alpha_{1}, \alpha_{2}$ are the indices below and above the break respectively for $\alpha_{1}<\alpha_{2}$, and the spectrum breaks around a momentum centred on $p_{b r}$; the parameter $\delta$ controls the sharpness of the break: smaller $\delta$ produces a sharper break, typical values are $\delta=0.5-1.5$. It converges to the given power-laws at low and high $p$, with a smooth transition. This is the same form as used for CR in supernova remnants in (17), written in a symmetrical form in the indices for clarity. This function has one more parameter $(\delta)$ than for a perfectly sharp break; it simply makes the break smoother in a controlled way. Given a reference value $n_{\text {ref }}$ at $p_{\text {ref }}$ the normalized spectrum is:

$$
n(p)=n_{r e f} \times\left[\left(\frac{p_{r e f}}{p_{b r}}\right)^{\alpha_{1} / \delta}+\left(\frac{p_{r e f}}{p_{b r}}\right)^{\alpha_{2} / \delta}\right]^{\delta} /\left[\left(\frac{p}{p_{b r}}\right)^{\alpha_{1} / \delta}+\left(\frac{p}{p_{b r}}\right)^{\alpha_{2} / \delta}\right]^{\delta}
$$

The normalization $n_{r e f}\left(p_{r e f}\right)$ is treated as a free parameter; there are thus 5 parameters each for protons and leptons, making 10 parameters in total. Since gamma rays cannot distinguish an origin in CR protons from CR helium, some assumptions have to be made to break the degeneracy. The ratio of protons and helium is fixed to that measured by PAMELA at $100 \mathrm{GeV}$, where solar modulation is absent while the experimental error is small. We take a reference proton momentum of $100 \mathrm{GeV}$, with a wide prior range since the normalization is to be determined from the gammaray data only. The lepton (electron + positron) spectrum is only marginally constrained by gamma rays, so we rely on direct measurements for the normalization and high-energy spectral index, allowing a range with uniform prior. We use the Fermi-LAT lepton flux at $20 \mathrm{GeV}$ (14). For the low-energy (below about $1 \mathrm{GeV}$ ) spectral index we rely on synchrotron radiation constraints as described later. The prior range for the break position is based on both synchrotron and direct measurements.

\section{Results and Discussion}

We illustrate the results using the raw emissivities accounting for energy dispersion; using the corrected values without applying dispersion leads to very similar results. The fit results are given in Table 1 and Fig 1 . The parameters are highly correlated so the individual values are just indicative, while the resulting spectrum error band (Fig 1) uses the full posterior in all parameters.

The proton spectrum spectral index above the break is recovered in good agreement with direct measurements (beyond the effects of solar modulation); note that it has been determined from the 
Table 1: Summary of model fits to equation 6.1. Entries are prior range, posterior mean and standard deviation. The proton parameters are constrained by the $\gamma$-ray emissivities, while the lepton parameters reflect mainly the prior from synchrotron and direct measurements. The parameters are highly correlated and degenerate, so the resulting spectrum derived from the full posterior (Fig 1) is preferred to the individual parameters. The CR density $n_{r e f}$ is multiplied by $(c / 4 \pi)$ to give a flux in the usual units quoted in experiments. $p_{\text {ref }}=10^{5} \mathrm{MeV}$ for protons, $2 \times 10^{4} \mathrm{MeV}$ for leptons.

\begin{tabular}{llllll}
\hline Parameter & range: $\min$ & $\max$ & mean & std & units \\
\hline Protons & & & & & \\
\hline$(c / 4 \pi) n_{\text {ref }}$ & $1 \times 10^{-9}$ & $20 \times 10^{-9}$ & $6.4 \times 10^{-9}$ & $0.3 \times 10^{-9}$ & $\mathrm{~cm}^{-2} \mathrm{sr}^{-1} \mathrm{~s}^{-1} \mathrm{MeV}^{-1}$ \\
$\alpha_{1}$ & 2.2 & 2.7 & 2.37 & 0.09 & \\
$\alpha_{2}$ & 2.6 & 3.5 & 2.82 & 0.05 & \\
$\delta$ & 0.05 & 1.0 & 0.5 & 0.1 & \\
$p_{b r}$ & 1000 & 10000 & 5870 & 2200 & $\mathrm{MeV}$ \\
\hline Leptons & & & & & \\
\hline$(c / 4 \pi) n_{\text {ref }}$ & $1 \times 10^{-9}$ & $3 \times 10^{-9}$ & $2.2 \times 10^{-9}$ & $0.5 \times 10^{-9}$ & $\mathrm{~cm}^{-2} \mathrm{sr}^{-1} \mathrm{~s}^{-1} \mathrm{MeV}^{-1}$ \\
$\alpha_{1}$ & 1.8 & 2.2 & 2.0 & 0.1 & \\
$\alpha_{2}$ & 3.1 & 3.2 & 3.15 & 0.03 & \\
$\delta$ & 0.05 & 1 & 0.47 & 0.25 & \\
$p_{b r}$ & 500 & 2000 & 1130 & 4067 & $\mathrm{MeV}$ \\
\hline
\end{tabular}

gamma-ray data alone. The spectral break is significant, the index being smaller by $\sim 0.5$ below the break; this is where solar modulation affects the direct measurements, as can clearly be seen in the difference between PAMELA, AMS01 and the interstellar spectrum. Note that we address a break in the momentum spectrum, not kinetic energy; the latter representation is not appropriate, see (1; 2). The overall normalization of the proton spectrum is about $30 \%$ larger than direct measurements, even in the high-energy region free from modulation. This can be a real physical effect since there is no guarantee that the direct measurements represent the average in the local region within one kpc probed by the gamma rays. There are spectral variations between the local region and the rest of the Galaxy $(18 ; 19)$, and the region probed by the emissivity analysis might be not so "local" in this sense. However there are sufficient uncertainties in the analysis to make such an interpretation premature at this stage: hadronic cross-sections have still significant uncertainties especially for CR and target nuclei with $A>1$. Uncertainties in the gas column densities including gas not traced by $\mathrm{HI}$ or $\mathrm{CO}$ are still significant, even though the emissivity derivation is for atomic hydrogen well traced by the $21-\mathrm{cm}$ line, correlations between the gas phases does not allow full separation of the components. Ionized hydrogen is accounted for in the analysis, but again cannot necessarily be fully separated from the other components. We note that (5) implicitly finds a similar trend in the normalization: that analysis included proton and helium direct measurements in the fitting so is more constrained to agree with those. In fact their proton spectrum is slightly above direct measurements, and their predicted emissivities are slightly below the measurements; so to reproduce the emissivities the proton spectrum would have to be increased further relative to direct measurements, in accord to what we find here. Different cross-sections and analysis techniques (see below) can account for any remaining differences. One important use of the interstellar spectrum is 

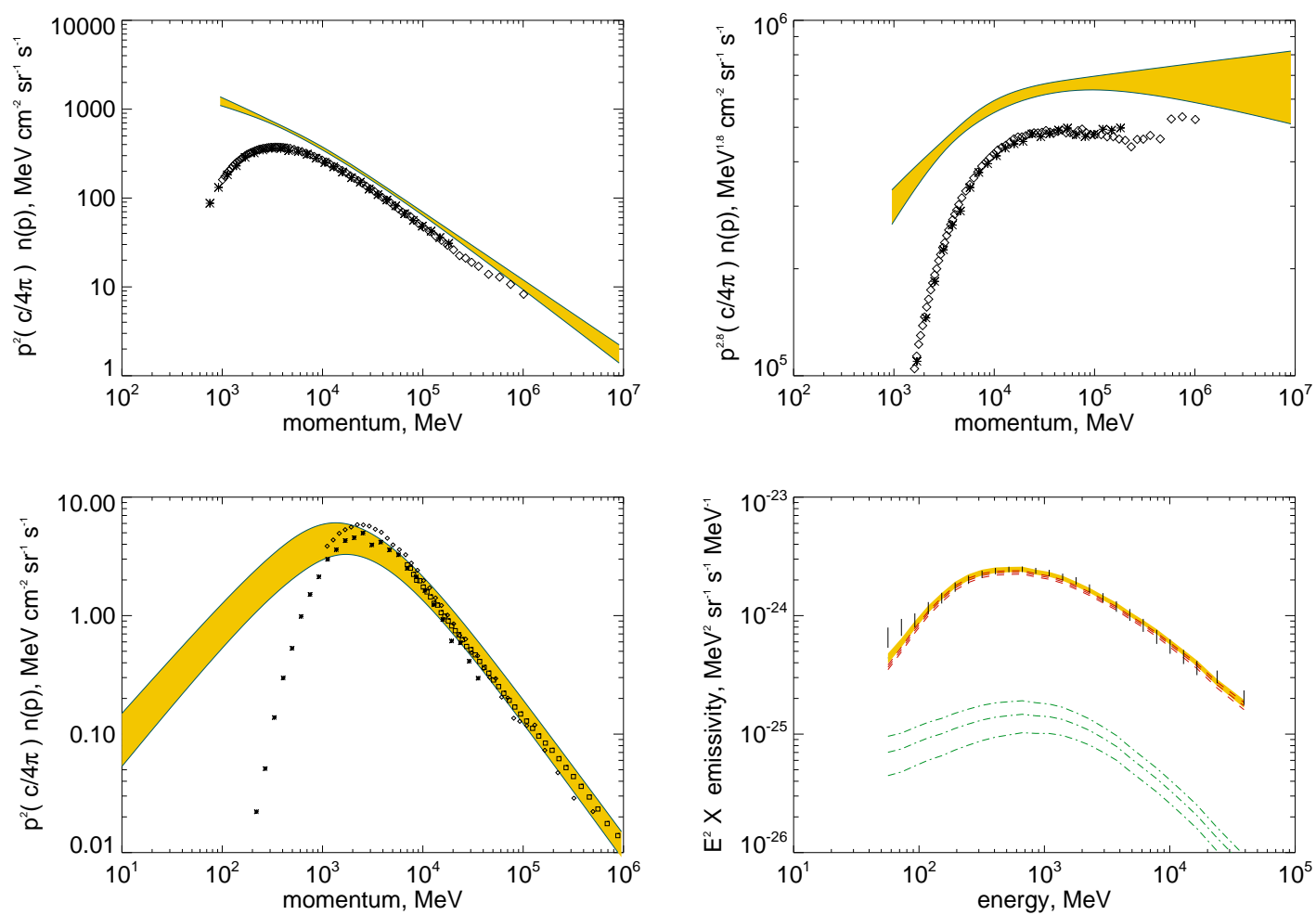

\section{PRELIMINARY}

Figure 1: Cosmic-ray and emissivity spectra derived from model fitting. Yellow band shows model range. Model ranges are 1 standard deviation on the parameterized synthetic spectra. Upper: Measured and derived cosmic-ray proton spectra. Data are AMS01 (asterisks) and PAMELA (diamonds). Spectra are multiplied by $p^{2}$, and also times $p^{2.8}$ to better show the break. Lower left: Measured and derived cosmic-ray electron spectra. Data are AMS01 (asterisks), PAMELA (diamonds), and Fermi-LAT (squares). The CR density $n(p)$ is multiplied by $(c / 4 \pi)$ to give a flux at high momenta in the usual units quoted in experiments. Lower right: Fermi-LAT emissivity data (vertical bars) and model, with red and green curves showing the hadronic and leptonic bremsstrahlung contributions; the yellow band shows the total.

for solar modulation studies; in this case it would be appropriate to use the spectral shape, which is well determined, but renormalize the spectrum to agree with direct measurements at high energies. This would attribute all the normalization difference to the factors mentioned above.

The interpretation of the curvature in the proton spectrum is beyond the present work, but it is generally consistent with expectation based on $\mathrm{CR}$ propagation implied by the $\mathrm{B} / \mathrm{C}$ ratio, which peaks at a few $\mathrm{GeV}$, implying either a break in the diffusion coefficient, effects of diffusive reacceleration, or convective transport. Any of these effects will cause a low-energy break in the primary spectra including that of protons, superimposed on a power-law injection spectrum (20). Note the apparent difference in index between the local proton spectrum and the large-scale Galaxy $(18 ; 19)$; the latter spectrum is evidently harder. The origin of the difference is not clear at present, but it is interesting that the index from local gamma rays and local direct measurements are consistent. There are further suggestions of local CR spectral variations (21). 


\section{Comparison of analyses}

The present analysis differs from that of (5) (Cas15) in various ways; both approaches are valid and are complementary, emphasizing different aspects of the subject. The main differences can be summarized as follows: Cas15 performs an iterative correction for energy dispersion, we include it in the response to the raw emissivities. Cas15 includes direct measurements of protons and helium in the fit, combined with the emissivities. We use only $\gamma$-ray data for the hadronic contribution, and adopt a He/p ratio from direct measurements, assuming equal spectral shapes for $\mathrm{p}$ and He. Cas 15 uses a velocity term to obtain the low-energy turnover in the proton spectrum; we use a smoothly broken power law for more flexibility. Cas15 uses a flux spectrum in kinetic energy, we use a density spectrum in momentum. Cas15 uses cross-sections from Kamae; we use a combination from Dermer and QGSJET-II. Cas15 uses a lepton spectrum from direct measurements combined with the $\gamma$-ray emissivities, we combine direct measurements with constraints from synchrotron. Cas15 uses Minuit for the fitting, we use a Bayesian scheme with MultiNest.

\section{Acknowledgments}

I thank Jean-Marc Casandjian for providing the emissivity data and useful discussions, and Chuck Dermer, Michael Kachelriess and Sergey Ostapchenko for their hadronic production software and many discussions about the physical processes.

The Fermi-LAT Collaboration acknowledges support for LAT development, operation and data analysis from NASA and DOE (United States), CEA/Irfu and IN2P3/CNRS (France), ASI and INFN (Italy), MEXT, KEK, and JAXA (Japan), and the K.A. Wallenberg Foundation, the Swedish Research Council and the National Space Board (Sweden). Science analysis support in the operations phase from INAF (Italy) and CNES (France) is also gratefully acknowledged.

\section{References}

[1] C. D. Dermer, A. W. Strong, E. Orlando, and L. Tibaldo ArXiv e-prints (July, 2013) [arXiv:1307.0497].

[2] C. D. Dermer, J. D. Finke, R. J. Murphy, A. W. Strong, F. Loparco, M. N. Mazziotta, E. Orlando, T. Kamae, L. Tibaldo, J. Cohen-Tanugi, M. Ackermann, T. Mizuno, and F. W. Stecker ArXiv e-prints (Mar., 2013) [arXiv: 1303.6482].

[3] J.-M. Casandjian Proc. 33rd ICRC, Paper ID 966 (2013).

[4] C. D. Dermer ArXiv e-prints (May, 2015) [arXiv: 1505.0575].

[5] J.-M. Casandjian ArXiv e-prints (May, 2015) [arXiv:1506.0004].

[6] F. Feroz, M. P. Hobson, and M. Bridges MNRAS 398 (Oct., 2009) 1601-1614, [arXiv:0809.3437].

[7] M. Kachelrieß and S. Ostapchenko PRD 86 (Aug., 2012) 043004, [arXiv: 1206.4705 ]. 
[8] M. Kachelriess, I. V. Moskalenko, and S. S. Ostapchenko ApJ 789 (July, 2014) 136, [arXiv: 1406.0035$]$.

[9] S. Ostapchenko vol. 52 of European Physical Journal Web of Conferences, p. 2001, June, 2013.

[10] K. Lodders ApJ 591 (July, 2003) 1220-1247.

[11] A. W. Strong, E. Orlando, and T. R. Jaffe $A \& A 534$ (Oct., 2011) A54, [arXiv:1108.4822].

[12] T. R. Jaffe, A. J. Banday, J. P. Leahy, S. Leach, and A. W. Strong MNRAS 416 (Sept., 2011) 1152-1162, [arXiv:1105.5885].

[13] E. Orlando and A. Strong MNRAS 436 (Dec., 2013) 2127-2142, [arXiv : 1309 . 2947 ].

[14] M. Ackermann, et al. PRD 82 (Nov., 2010) 092004-+.

[15] M. Ackermann et al. Physical Review Letters 108 (Jan., 2012) 011103, [arXiv:1109.0521].

[16] Planck Collaboration ArXiv e-prints (June, 2015) [arXiv:1506.0666].

[17] M. Ackermann et al. Science 339 (Feb., 2013) 807-811, [arXiv:1302 . 3307].

[18] D. Gaggero, A. Urbano, M. Valli, and P. Ullio PRD 91 (Apr., 2015) 083012, [arXiv:1411.7623].

[19] A. Neronov and D. Malyshev ArXiv e-prints (May, 2015) [arXiv:1505. 0760].

[20] A. W. Strong, I. V. Moskalenko, and V. S. Ptuskin Annual Review of Nuclear and Particle Science 57 (2007) 285-327.

[21] M. Kachelriess, A. Neronov, and D. V. Semikoz ArXiv e-prints (Apr., 2015) [arXiv:1504.0647]. 